\title{
Potential Role of Blood Biomarkers in the Management of Nontraumatic Intracerebral Hemorrhage
}

\author{
Rebecca Senn $^{a}$ Mitchell S.V. Elkind ${ }^{c}$ Joan Montaner ${ }^{b}$ Mirjam Christ-Crain ${ }^{a}$ \\ Mira Katan ${ }^{d}$ \\ ${ }^{a}$ Department of Endocrinology, University Hospital Basel, Basel, Switzerland; ${ }^{b}$ Department of Neurology, \\ Val d'Hebron, Barcelona, Spain; ' Departments of Neurology and Epidemiology, Columbia University, \\ New York, N.Y., USA; d Department of Neurology, University Hospital of Zurich, Zurich, Switzerland
}

\section{Key Words}

Blood biomarker · Intracerebral hemorrhage .

Management $\cdot$ Stroke

\begin{abstract}
Background: Intracerebral hemorrhage (ICH), a subtype of stroke associated with high mortality and disability, accounts for $13 \%$ of all strokes. Basic and clinical research has contributed to our understanding of the complex pathophysiology of neuronal injury in $\mathrm{ICH}$. Outcome rates, however, remain stable, and questions regarding acute management of $\mathrm{ICH}$ remain unanswered. Newer research is aiming at matching measured levels of serum proteins, enzymes, or cells to different stages of brain damage, suggesting that blood biomarkers may assist in acute diagnosis, therapeutic decisions, and prognostication. This paper provides an overview on the most promising blood biomarkers and their potential role in the diagnosis and management of spontaneous ICH. Summary: Information was collected from studies, reviews, and guidelines listed in PubMed up to November 2013 on blood biomarkers of nontraumatic ICH in humans. We describe the potential role and limitations of GFAP, S100B/RAGE, and ApoC-III as diagnostic biomarkers, $\beta$-Amyloid as a biomarker for etiological classification, and 27 bio-
\end{abstract}

markers for prognosis of mortality and functional outcome. Within the group of prognostic markers we discuss markers involved in coagulation processes (e.g., D-Dimers), neuroendocrine markers (e.g., copeptin), systemic metabolic markers (e.g., blood glucose levels), markers of inflammation (e.g., IL6), as well as growth factors (e.g., VEGF), and others (e.g., glutamate). Some of those blood biomarkers are agents of pathologic processes associated with hemorrhagic stroke but also other diseases, whereas others play more distinct pathophysiological roles and help in understanding the basic mechanisms of brain damage and/or recovery in $\mathrm{ICH}$. Key Messages: Numerous blood biomarkers are associated with different pathophysiological pathways in $\mathrm{ICH}$, and some of them promise to be useful in the management of $\mathrm{ICH}$, eventually contributing additional information to current tools for diagnosis, therapy monitoring, risk stratification, or intervention. Up to date, however, no blood biomarker of $\mathrm{ICH}$ has been studied sufficiently to find its way into clinical routine yet; well-designed, large-scale, clinical studies addressing relevant clinical questions are needed. We suggest that the effectiveness of biomarker research in ICH might be improved by international cooperation and shared resources for large validation studies, such as provided by the consortium on stroke biomarker research (http://stroke-biomarkers.com/page.php?title=Resources).

(c) 2014 S. Karger AG, Basel

Mira Katan, MD, MS

University Hospital of Zurich, Department of Neurology

Frauenklinikstrasse 26

CH-8091 Zurich (Switzerland)

E-Mail Mira.Katan@usz.ch 


\section{Introduction}

\section{Epidemiology of ICH}

Stroke annually brings 15 million patients into emergency rooms worldwide, of which 5.5 million die and another 5 million are left permanently disabled [1]. Stroke is primarily categorized into ischemic (approximately $87 \%$ ) and hemorrhagic (approximately 13\%, including subarachnoid hemorrhages) stroke [2].

Spontaneous intracerebral hemorrhage (ICH) is associated with poorer outcome compared to ischemic stroke (IS), with 30-day mortality rates of 37-52\% [2] and only $20 \%$ of survivors returning to independent function at 6 months [3]. These rates have not changed over the past 20 years [4].

While high blood pressure used to account for $80-90 \%$ of ICH, today this number has decreased to $50 \%$, presumably as a result of efficient hypertension management [5] and an increasing accuracy in the diagnosis of other etiologies because computed tomography (CT) and especially magnetic resonance imaging (MRI) are more widely available. The gap therefore is being filled up by an increasing number of long-term anticoagulation-related $\mathrm{ICH}$ (17\% of ICH) [6], for example, on the basis of vascular malformations, and especially cerebral microbleeds [7], which are generally associated with larger hemorrhage size and higher mortality. The third etiological subcategory, accounting for $20 \%$ of $\mathrm{ICH}$, is the deposition of amyloid protein in cerebral arteries (cerebral amyloid angiopathy, CAA), causing the blood vessels to leak more easily [5].

\section{Pathophysiology of ICH}

Advances in neuroimaging and animal models have improved our understanding of the pathophysiology of ICH on macro-, molecular, and genetic levels [8]. By analyzing the cascade of brain damage after ICH, targets for new therapies and future biomarkers can be identified. Most current theories imply a two-phase model of neuronal injury with an early mechanical phase and a subacute phase of inflammation and edema formation as response to the hemorrhage [9], as well as the parallel activation of the neuroendocrine stress axis.

\section{Primary Phase: Mechanical Damage}

Within $60 \mathrm{~min}$ after the insult, the blood released into the brain parenchyma leads to the compression of anatomical structures and increased intracranial pressure, potentially leading to brain herniation [10], decreased blood flow, and direct mechanical destruction of axons and glial cells [11] (fig. 1).
Secondary Phase: Physiological Response to

Hematoma

The body reacts to the bleeding and rise of intracranial pressure with different parallel mechanisms that accelerate themselves and each other, increasingly compounding the neurological injury (fig. 2).

One of the first responses to acute bleeding is the stress-related activation of the hypothalamo-pituitary adrenal (HPA)-axis as well as the sympathetic nervous system (SNS) [12], both of which act systemically. Locally, glutamate is released by necrotic perihematomal neurons and by the ruptured vessels, leading to the accumulation of oxidative byproducts, further necrosis of neighboring cells, and early cytotoxic edema formation [13].

Within the first two days, hemostatic mechanisms occur to limit the bleeding. Thrombin is released and affects a range of cells in the surrounding penumbra, creating inflammatory, neurotoxic conditions. Thrombin activates microglial cells to release inflammatory mediators, causes proliferation of mesenchymal cells and formation of scar tissue, and leads to endothelial cell dysfunction, resulting in hyperpermeability, disruption of blood brain barrier (BBB), and vasogenic edema formation [14].

After several days, iron, hemoglobin, and free radicals are released into the hemorrhagic area, following the lysis of erythrocytes and clot components as part of the thrombin response, causing further inflammation and late edema formation [15].

The last mechanism resulting from intracerebral inflammation and late edema is the activation of the complement system. As C5a and C3a penetrate the neuronal tissue, local glia, mast cells, and leukocytes are activated, followed by the additional damage of perihematomal neurons, glia, and blood vessels, and further cell lysis and inflammation [16].

\section{Management of ICH}

Modern ICH management including accurate diagnosis, effective treatment, prediction of progression, response to therapy, and prognostication remains challenging. Management decisions are mainly based on neuroimaging information such as hematoma volume, location, and the presence and quantity of intraventricular bleeding, and clinical information such as age and the Glasgow coma scale (GCS). These features are all represented in the Hemphill ICH score [17]. As ICH is a medical emergency, it would be beneficial to have a diagnostic tool applicable in pre-hospital diagnosis, early sub-typing, and risk stratification. Blood biomarker (BBM) research may be helpful. 
Fig. 1. Pathophysiology of ICH Primary Phase: Mechanical Brain Damage.

Fig. 2. Pathophysiology of ICH Secondary Phase: Physiological Response to Hematoma.
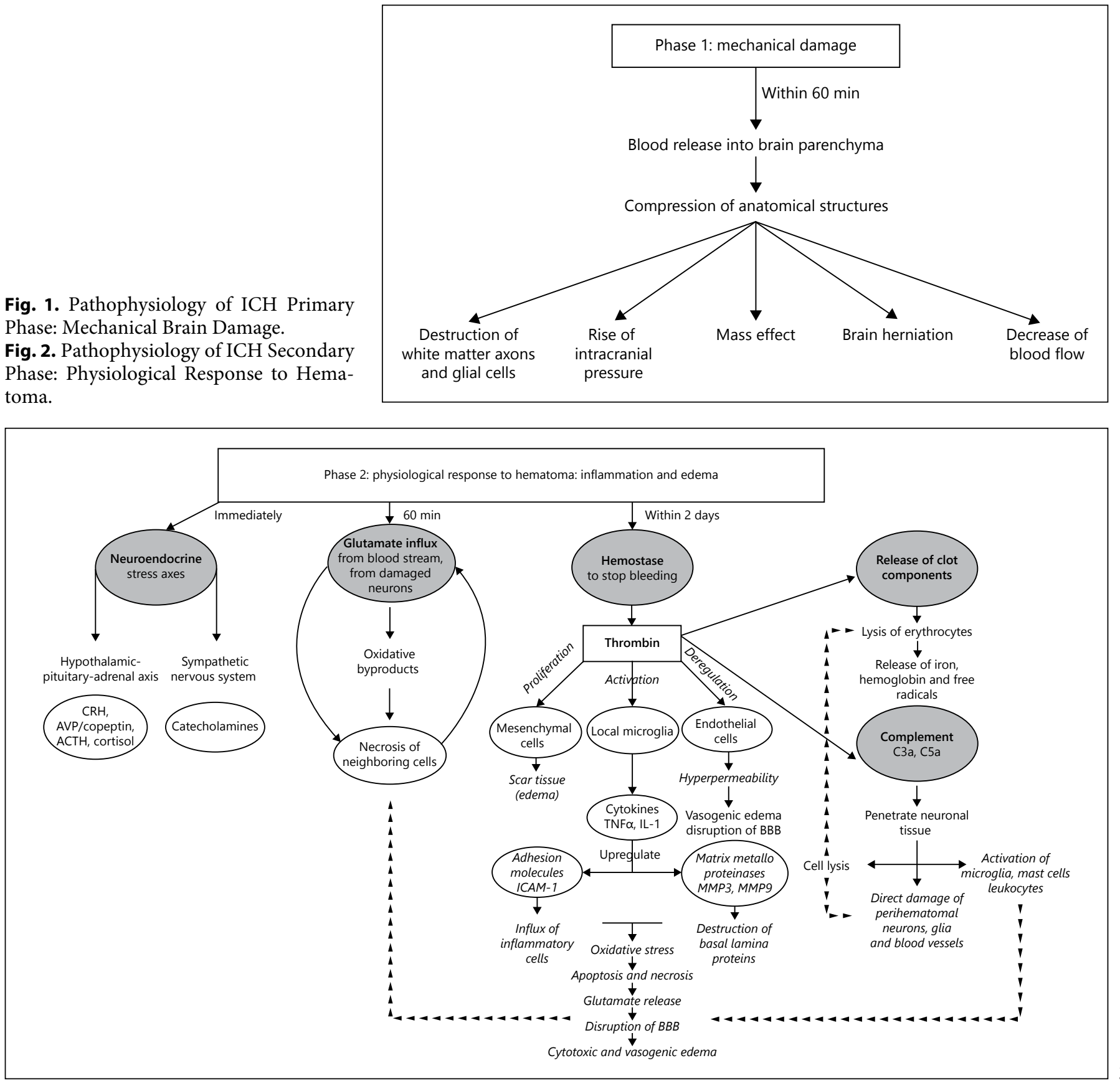

\section{Definition and Criteria for Novel Blood Biomarkers}

BBMs are measurable products reflecting healthy or pathologic processes of the body. The ideal measurement of a BBM should be simple, quick, and preferably inexpensive. Therefore, a BBM should be easily measurable in accessible tissue, reproducible, and relatively stable throughout the healthy population. Depending on the use of the BBM, different challenges need to be addressed, one of the most important being that these BBMs add information to already accessible clinical or imaging information [18].

\section{Identification and Selection of Blood Biomarkers in $\mathrm{ICH}$}

The classical evaluation of new candidate BBMs - and emphasis of this review - is based on pre-specified hy- 
potheses, searching pathophysiologic mechanisms of ICH for in blood measurable, involved agents.

Newer approaches based on genetic and molecular research are fairly unbiased discovery processes. Gene expression profiling has generated diverse insights on RNA expression and protein and metabolite production, depicting changes in expression, induction, and function of genes in individuals with stroke. The advantage of such approaches is that they generate a number of potential markers of interest, but the difficulty is that the utility of each individual marker remains uncertain until further studies can validate the results [19]. Proteomics and metabolomics are new techniques to systematically scan blood from cases and controls for differentially expressed proteins and molecules (lipids, sugars, nucleotides, amino acids), potentially revealing new disease-related particles [20]. These methods, in opposition to genetic profiling, respond more strongly to environmental influences and thus are dynamic in reflecting current physiologic states [21]. Genetic and molecular technologies belong to the very first discovery phase of BBM research as an enormous flood of information is generated, which needs versatile elaborate analysis to select those agents possibly meaningful for further studies.

While we recognize that cerebrospinal fluid biomarkers may have certain advantages over blood biomarkers, we focus this review on blood biomarkers alone because they are more readily measured repeatedly; there are a large number of studies addressing blood biomarkers, and space is limited.

\section{Search Methods}

A comprehensive literature search was conducted on PubMed up to November 2013, including citation and reference tracking to identify the blood biomarkers cited in this review. The search terms employed were: ' $\mathrm{ICH}$ ', 'intracerebral h(a)emorrhage/h(a)ematoma', 'h(a)emorrhagic stroke' + 'biomarker(s)' + 'management', 'outcome', 'prognosis', 'diagnosis', and 'etiology'. With this retrieval strategy a total of 186 manuscripts was yielded.

\section{Clinical Implementation of ICH Blood Biomarkers}

\section{Diagnostic Blood Biomarkers}

Diagnostic BBMs should be able to reliably distinguish between patients suffering from ICH, IS, and stroke mimics such as seizures, migraines, syncope, or metabolic conditions [22], enabling rapid medical interventions. This information is required shortly after symptom onset be- cause early neurological damage within the first few hours after stroke is strongly associated with poor long-term outcome [23]. BBMs differentiating ICH from IS already in the ambulance might be especially useful to start blood pressure lowering therapies in a very early stage (as suggested by the results of the INTERACT2 trial [24]).

\section{Etiologic Blood Biomarkers}

Etiologic BBMs should allow differentiation between bleeding based on hypertension, long-term anticoagulation, CAA or other more infrequent causes. Such BBMs could increase the quality of rapid specific treatment, which differs according to the pathogenesis (blood pressure control, immediate cessation of anticoagulant therapy, hemostatic therapy depending on the drug used, and/or surgical hematoma evacuation). Etiologic BBMs should be found in blood sampled at admission as only early information can lead to early therapy.

\section{Blood Biomarkers of Prognosis}

Prognostic BBMs have the potential to predict endpoints like complications, mortality, or poor functional outcome, and therefore may enable preventive and therapeutic interventions [25]. Moreover, to later allow the integration of a BBM into the package of clinical instruments, its ability to depict specific causes of worsening (e.g., infection, pneumonia, epileptic seizures, hematoma growth), and prognosis-related situations that allow specific intervention, is crucial. For example, early time to surgery (excluding emergency decompression) was shown to improve mortality rates but also increase the risk of rebleeding. Sixty percent of ICH patients are at a low risk for further bleeding, and might therefore qualify for surgery, whereas patients with a high risk of rebleeding might benefit more from medical intervention only [26-28]. A BBM reliably discriminating the low risk from the high risk-rebleeding group would be very useful for the intervention triage. Depending on the time-point of blood withdrawal, prognostic BBMs could also enable early risk stratification, emergency health care decisions, and late interventions, such as rehabilitation and followup care. As prognostic BBMs correlate in an either protective or damaging way with disease progression, they might also help in identifying new therapeutic targets.

\section{Blood Biomarkers as Surrogates}

Surrogate markers are markers that substitute for true end points. This correlation needs to show a robust stability across different beneficial as well as aggravating interventions and alterations of circumstances, before a change 
of the biomarker might actually reliably predict the clinical outcome (in place of the clinical outcome itself) and therefore account as surrogate [29]. This high bar is set to prevent misleading information regarding the true end point by a 'surrogate' and is a tall order for any blood test. None of the BBMs mentioned below serves as such a good marker of change in disease status that it could be used in place of other measures (yet).

The most advanced BBM examples for each category are summarized in table 1.

\section{Selection of Blood Biomarkers in ICH}

\section{Blood Biomarkers for Diagnosis \\ GFAP}

Glial fibrillary acidic protein (GFAP) is an intermediate filament expressed by astrocytes and ependymal cells. After ICH it can be found in serum of patients due to necrotic brain cell destruction and BBB disruption. Low concentrations of GFAP are found in the blood of healthy controls; [30] it is increased after IS, but the highest concentrations are found in early ICH patients [31].

In a recent multicenter cohort study including 205 patients diagnosed with either IS, ICH, or stroke mimics, plasma GFAP on admission had a sensitivity of $84 \%$ and specificity of $96 \%$ for differentiating ICH from IS and stroke mimics (AUC 0.915, 95\% CI 0.847-0.982) [32].

Since this study only included patients with hemispheric stroke symptoms, the results cannot be generalized to all ICH patients. This also may have led to a higher sensitivity. Furthermore, only few mimics were included, potentially causing artificial increase of specificity.

\section{S100B/RAGE}

The transmembrane Receptor for Advanced Glycation Endproducts (RAGE) is a multiligand member of the immunoglobulin superfamily, which plays a major role in both early development and progression of atherosclerosis and vascular inflammation [33]. S100B is a brain-specific, calcium-binding protein and ligand for RAGE, expressed and released mainly by astrocytes [34]. S100B/ RAGE interactions might show different expression courses in inflammatory or hypoxic brain damage.

A large study recently showed the ability of the S100B/ RAGE BBM panel to distinguish between IS and ICH in a sample of 915 stroke patients (AUC 0.76) [35]. In blood sampled within $6 \mathrm{~h}$ from symptom onset, S100B was elevated, whereas sRAGE was lower in ICH patients compared to patients with IS. The additive value of the panel

Blood Biomarkers in Intracerebral Hemorrhage to the AUC when added to the model using only clinical signs was significant (AUC 0.84 vs. AUC 0.77) and even stronger when measured earlier $(<3 \mathrm{~h}$ from stroke; AUC 0.81 vs. AUC 0.76) [35].

Although promising, the dynamics of sRAGE in stroke is not yet clearly understood. The results need validation in larger cohort studies including different settings, stroke mimics, and additional BBMs.

ApoC- III

ApoC-III is a member of the apolipoprotein family expressed by the liver. It is found mainly in very-low-density lipoprotein (VLDL) particles and is associated with cardiovascular risk.

In a small case-control study that measured ApoC-III expression in stroke patients within $6 \mathrm{~h}$ from symptom onset, a relative overexpression of ApoC-III was found in IS compared to ICH patients. ApoC-III levels allowed differentiation of ICH from IS with 94 sensitivity and $87 \%$ specificity and distinguished ICH from healthy controls with $100 \%$ sensitivity and $67 \%$ specificity [36].

So far, ApoC-III seems to be an interesting marker to distinguish ICH from IS, but the sample size was small and data was not collected prospectively. A larger validation study should also include stroke mimics.

\section{Blood Biomarkers for Etiological Classification}

$\beta$-Amyloid

$\beta$-Amyloid $(\beta A)$ is a protein pathologically deposited in smaller arteries of the brain of patients suffering from CAA, causing them to leak or rupture more easily [37].

By comparing plasma of CAA-related $\mathrm{ICH}$ patients to healthy controls, it was shown that $\beta A$ was significantly higher in CAA cases than in controls. $\beta$ A levels even differentiated between patients with probable CAA and those diagnosed with possible CAA $(p<0.015)$ or controls $(p<0.005)[38]$. The development of a $\beta A$ screening method for CAA would be particularly useful, as diagnosis of CAA prior to hemorrhage is not yet possible [39]. Confirmation in a prospective multicenter study is indispensable, as the sample size was small and a previous study showed contradicting results [40].

\section{Blood Biomarkers for Prognosis and as \\ Pharmacological Targets \\ MMP-9}

Matrix metalloproteinase 9 (MMP-9) is a protease induced by thrombin and blood, which increases capillary permeability, disrupts $\mathrm{BBB}$, and is neurotoxic by degrading the endothelial basal lamina and extracellular 


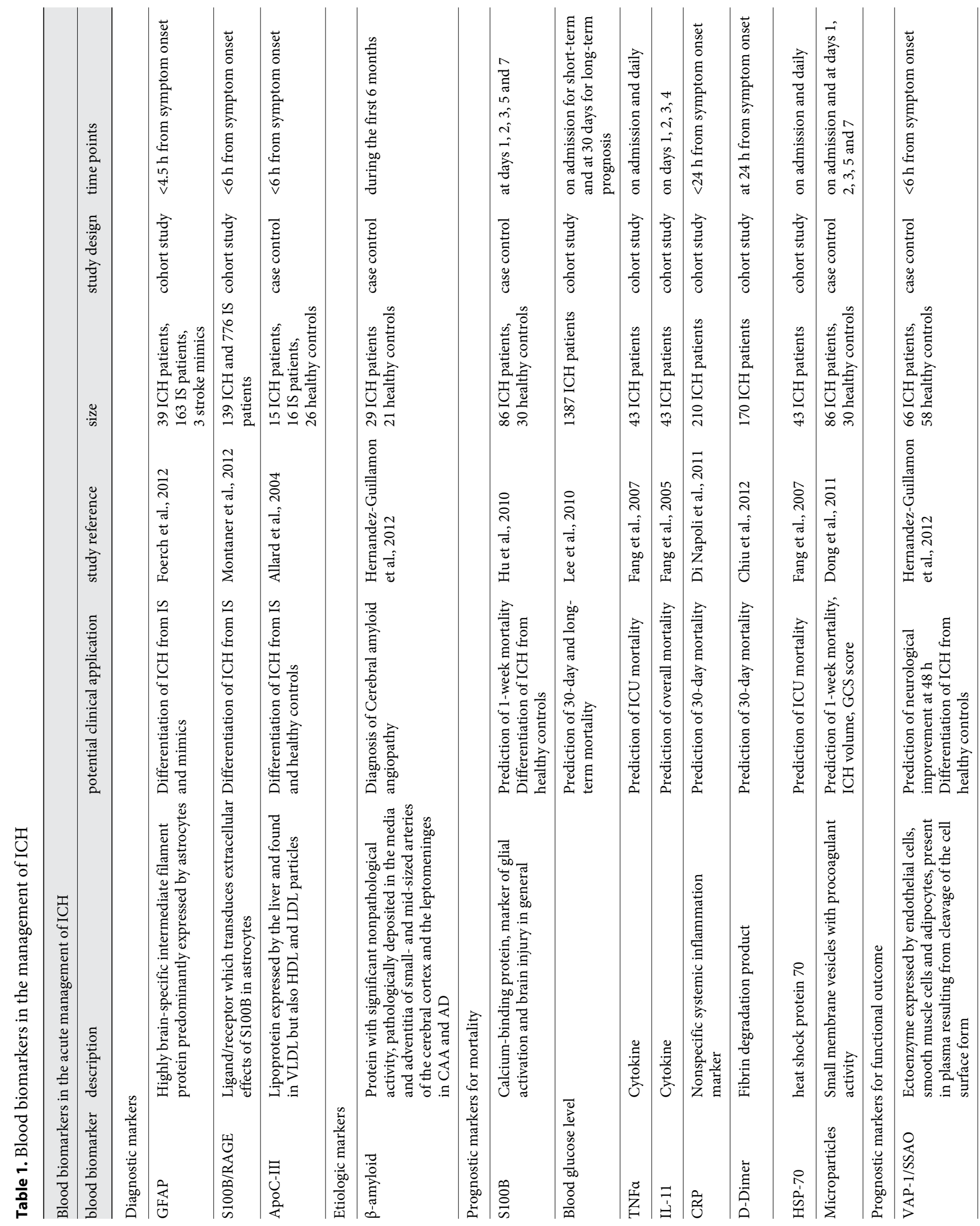




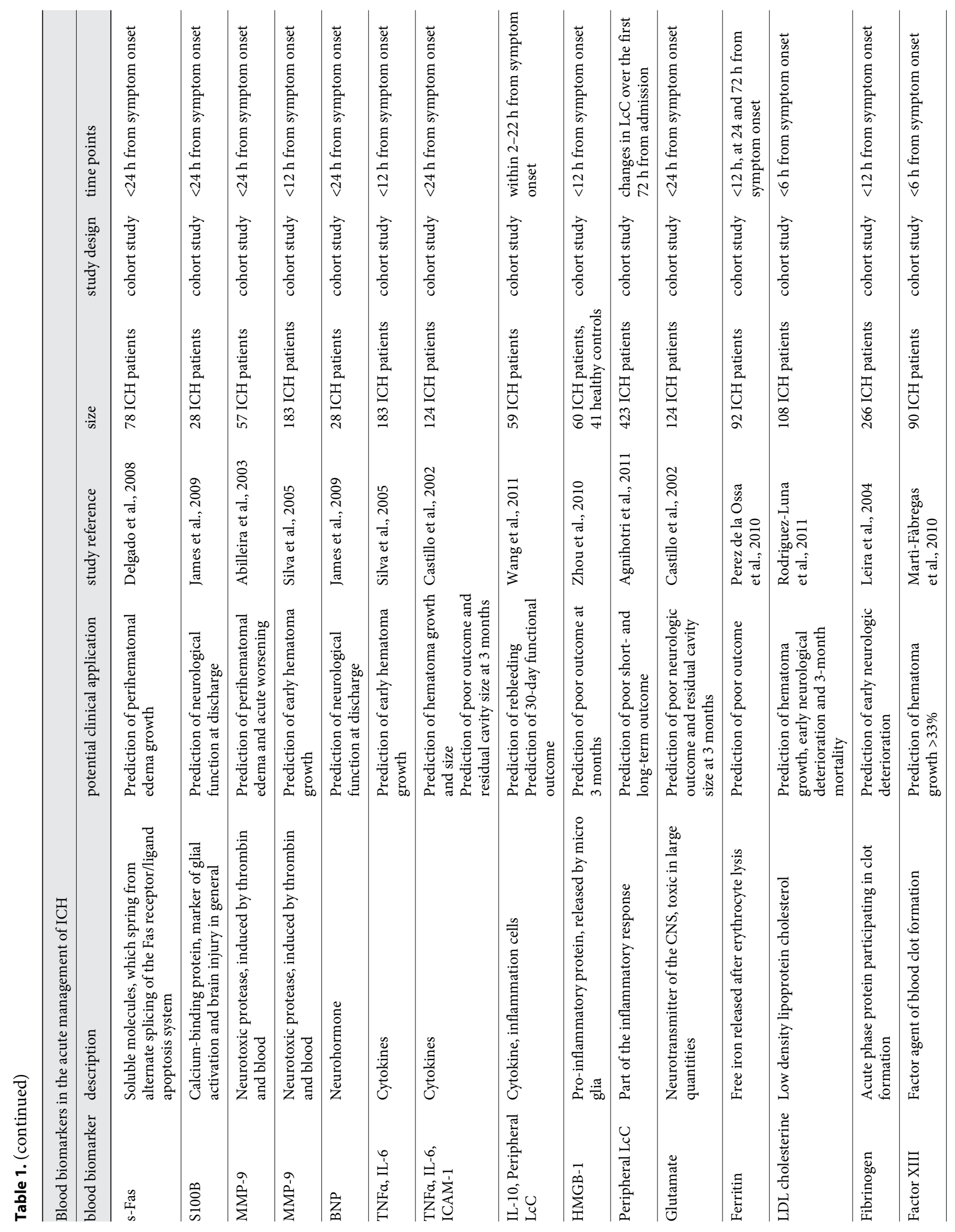




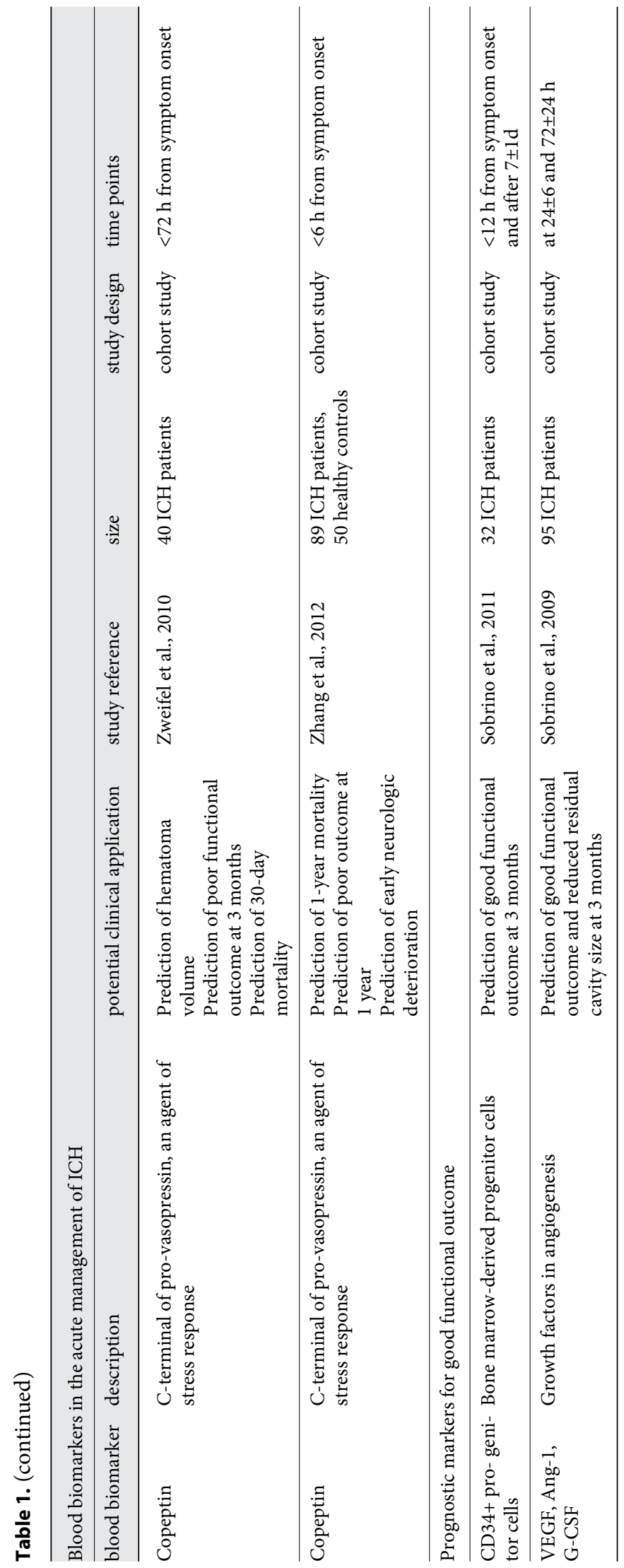

matrix $[41,42]$. On the other hand, MMP-9 contributes in beneficial ways by promoting angiogenesis, remodeling, cell migration, and phagocytosis [43].

In a small cohort study, MMP-9 was found to be elevated after ICH [44]. In cases of deep bleeding, MMP-9 is significantly correlated with edema size and neurological worsening. Another larger cohort study found high MMP-9 levels correlate with a percentage of hematoma growth $(\mathrm{r}=0.64)$ [45].

MMP-9 seems to play an important role in complications after ICH [46]. Its role as a predictor of hematoma growth needs validation in larger studies. If validated, MMP-9 may play a role in selecting patients for trials investigating hemicraniectomy in $\mathrm{ICH}$.

\section{BNP}

B-type natriuretic peptide (BNP) is a hormone secreted by the smooth muscle cells of the heart when stretched [47]. Recent research [48] suggests that BNP plays a role in disease progression of neuronal injuries including ICH.

In a small cohort study, BNP levels correlated with the ICH score $(r=0.42)$ and independently predicted outcome at discharge [49].

The mechanisms by which endogenous high BNP levels are related to disease progression after $\mathrm{ICH}$ are not yet understood and require further research.

\section{VAP-1/SSAO}

Vascular adhesion protein-1 (VAP-1) belongs to the semicarbazide-sensitive amine oxidase (SSAO) family, enzymes involved in the process of immune cell migration, and found either on the cell surface or soluble in serum.

In ICH patients, low VAP-1 independently predicted neurological improvement after $48 \mathrm{~h}(\mathrm{OR}=6.8)$ after accounting for baseline stroke characteristics [50]. This supports the finding that inhibition of VAP-1 activity in mice reduced brain cell destruction [51].

VAP-1 shows potential as an outcome predictor, which needs to be verified in future studies also including stroke mimics. Moreover, comparing VAP-1 activities with other important predictors of outcome, such as ICH volume and early hematoma growth, are required.

\section{s-Fas}

Soluble Fas (s-Fas) are variant molecular splicing products of the Fas-receptor/ligand apoptosis signaling pathway, and they belong to the tumor necrosis factor (TNF) receptor family. Some s-Fas molecules were found to be potential inhibitors of the Fas-apoptosis system, and appear to correlate with neuronal cell death [52]. 
It was shown that s-Fas is significantly lower in $\mathrm{ICH}$ patients than in healthy controls, when blood is withdrawn within $24 \mathrm{~h}$ from symptom onset. Moreover, low baseline s-Fas proved to be an independent predictor for perihematomal edema growth $(\mathrm{OR}=0.125)$ [53]. Administration of s-Fas [54] inhibits the Fas-receptor/ligand system in a cell model [55], reducing neuronal damage.

These findings should further be elucidated, especially the role of the Fas system in cell death after ICH.

\section{S100B}

By inducing neuronal death, S100B stimulates inflammatory stress as well as dispersal of interleukins (IL-1 [56], IL-6) and TNFa [57] and therefore might correlate with disease progression.

In a small case-control study, plasma S100B was found to be significantly increased from symptom onset until day 3 in patients with ICH compared to healthy controls. It was an independent predictor for 1-week mortality [58]. Similarly, another study showed S100B to be an independent predictor for neurological functional outcome at discharge in a small cohort of ICH patients [49]. The

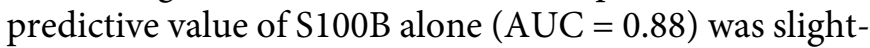
ly lower compared to areas under the curve of GCS scores $(\mathrm{AUC}=0.94)$ and $\mathrm{ICH}$ volume $(\mathrm{AUC}=0.93)$ [58].

There is a potential use of $\mathrm{S} 100 \mathrm{~B}$ as an outcome BBM but it needs verification in larger prospective cohort studies. Moreover, if S100B does not provide incremental value for prognostication in $\mathrm{ICH}$, it might not find its way into clinical routine.

\section{Blood Glucose Level}

A large-scale cohort study showed a significant relationship between blood glucose levels (BGL) on admission and 30-day mortality. Even after excluding patients with diabetes, BGL proved an independent risk factor for death (adjusted HR $=1.10$, 95\% CI 1.01-1.19). BGL remeasured at 30 days revealed increase in long-term mortality with each glucose quartile [59].

These results imply the importance for early BGL monitoring in ICH patients, but need to be taken carefully as management of glucose-levels has been proven delicate in other critically ill patients [60]. Early intervention studies need to evaluate the ideal range for glucose levels after ICH.

\section{Inflammatory Markers}

As part of the inflammatory response, cytokines (TNFa, IL-6, IL-10, and IL-11), acute phase proteins (CRP), immune cells (peripheral leukocytes), ICAM (a member of the immunoglobulin superfamily), and HighMobility-Group-Protein B1 (HMGB1, is a pro-inflammatory, DNA-binding protein released by micro glia [61]) play variable roles in the progress of ICH, being involved in the rupture of vessels, further bleeding and necrosis, but in the subsequent course also acting to promote recovery. Consequently, many of them correlate with severity of stroke in the acute phase.

$T N F \alpha, I C A M-1, I L-6, I L-10, I L-11$. In a small cohort, increased plasma TNFa on day 1 was found to predict intensive care unit (ICU) mortality [62]. High TNFa and IL-6 levels both proved independent predictors for early hematoma growth in $\mathrm{ICH}$ patients and were associated with increased risk of mortality and poor functional outcome at 3 months [45]. Another cohort study showed that TNFa, IL-6 and intercellular adhesion molecule 1 (ICAM1) independently correlated with hypodensity on CT at days 3-4. In this study, ICAM-1 was also shown to correlate with poor outcome at 3 months and with residual cavity size [63]. In a prospective study enrolling 59 consecutive ICH patients, IL-10 predicted rebleeding with $75 \%$ sensitivity and $72 \%$ specificity and independently predicted poor 30 -day functional outcome with $73 \%$ sensitivity and specificity of $66 \%$ [64]. Serum IL-11 was found to be significantly higher in nonsurvivors of a small ICH cohort on days 2-4 [65].

$C R P$. In a large ICH cohort, elevated blood C-reactive protein (CRP) was independently associated with increased 30-day mortality after ICH and even added an $8 \%$ improvement to the accuracy of the Hemphill score model [66].

$H M G B-1$. In a small case-control study, comparing ICH patients to healthy controls, higher plasma HMGB-1 levels measured within the first $12 \mathrm{~h}$ were associated with poor outcome. The study also showed significant correlation of HMGB-1 with levels of IL- 6 and TNF $\alpha$ and NIHSS $(\mathrm{r}=0.845)$ score at day 10 [67].

Peripheral Leukocyte Count. In a case-control study, the change of peripheral leukocyte count ( $\mathrm{LCC}$ ) was examined by measuring the difference between $\mathrm{LcC}$ on admission and the highest $\mathrm{LcC}$ in the first $72 \mathrm{~h}$. The study showed significant correlation between LcC and poorer discharge disposition ( $\mathrm{OR}=1.258$ ) as well as decline in modified Barthel index (MBI) at three months $(r=0.222)$ [3]. Another prospective study showed higher $\mathrm{LcC}$ in patients rebleeding within 30 days [64].

The mechanisms of brain tissue damage from blood vessel rupture, disruption of the $\mathrm{BBB}$, edema formation, and activation of the inflammatory response system are not yet completely understood. Inflammation may con- 
tribute to neurological damage in the beginning but contribute to recovery in late ICH. Larger cohort study designs are necessary, especially for usage in clinical prognostic decisions, to validate these findings over the long-term as these markers follow a dynamic course.

\section{CD34+ Progenitor Cells, VEGF, Ang-1, G-CSF}

CD34+ progenitor cells (CD34) in the blood are circulating ancestors of adult endothelial cells and play an important role in angiogenesis. Vascular endothelial growth factor (VEGF), Angiopoietin 1 (Ang-1) and granulocytecolony stimulating factor (G-CSF) are growth factors (GF) also participating in neoangiogenesis. Assuming a connection between effective neurovascularization and good recovery from hemorrhage, they were evaluated as potential prognostic markers.

CD34. In blood sampled from $32 \mathrm{ICH}$ patients within $12 \mathrm{~h}$ from symptom onset and on day 7, CD34 levels at day 7 but not day 1 positively correlated with good functional outcome $(\mathrm{OR}=1.17)$ and negatively with residual cavity volume at 3 months $(r=-0.607)$ [68]. A strong correlation was also found between CD34 levels and GFs at day 7.

VEGF, Ang-1, G-CSF. In a previous cohort study measuring GF levels of 95 patients with primary ICH on day 3, independent associations between VEGF, Ang-1 and G-CSF and neurologic improvement $(\mathrm{OR}=11.2 ; 14.7$ and 19.6, respectively) as well as reduced residual cavity size at 3 months were demonstrated [69].

The results show that CD34 and GFs are not markers usable in the acute phase of $\mathrm{ICH}$. But as their increase following ICH may be associated with a better functional outcome, further investigation of long-term dynamics of CD34 and GFs and their role in the healing process is warranted.

\section{Glutamate}

Glutamate is an important neurotransmitter of the healthy CNS and is pathologically released in excitotoxic quantities by necrotic astrocytes as they appear in ICH. A correlation with secondary damage after ICH is expected.

Glutamate was shown to independently predict poor neurologic outcome at 3 months and to positively correlate with residual cavity size in a cohort of $124 \mathrm{ICH}$ patients $(\mathrm{r}=0.846)$ [63].

As glutamate has been well studied in other contexts, it might offer treatment opportunities after ICH, by inhibition of its excitotoxic signaling pathways. Such speculations and its prognostic value need to be confirmed in larger intervention studies.

\section{Ferritin}

Free ferritin in the brain occurs in line with hemolysis of the escaped erythrocytes after ICH. The release of iron is assumed to lead to delayed brain edema formation by harming neuronal tissue via oxidative stress, glutamate release, and inflammatory response $[8,70]$.

In a prospective study, an independent association between free serum levels of ferritin on admission and poor outcome has been shown (stroke severity, ICH, and edema volumes all $r>0.60$ ) [70]. No correlation to other acute phase proteins was found, even after adjustment for signatures for acute inflammation. The authors therefore suggest that free baseline ferritin affects outcome directly, and they propose a link to the therapeutic opportunity of iron chelation, which however needs further investigation.

\section{LDL-Cholesterol}

Low-density lipoprotein (LDL) is involved in the transportation of lipid molecules (e.g., cholesterol). Although high levels are a risk factor for cardiovascular events, after stroke, low LDL and total cholesterol levels are associated with unfavorable outcomes. It may be that serum cholesterol is needed for the integrity of vessel walls and that lower levels decrease platelet aggregation thus predisposing for ICH growth [71].

A recent cohort study demonstrated an independent correlation between low LDL levels and hematoma growth at $24 \mathrm{~h}(\mathrm{OR}=4.24)$, and between early neurological deterioration $(\mathrm{OR}=8.27)$ and mortality at 3 months $(\mathrm{OR}=6.34)$. In this study, pretreatment with statin did not seem to have an influence [71]. In earlier studies, it was shown that low LDL-cholesterol and low total-cholesterol levels predict a higher risk for mortality after ICH [72-74].

Although LDL seems to be a strong and independent predictor, the study does not provide information on the additive value compared to other prognostic scores. There is a risk for selection bias due to exclusion of patients who were either comatose, who died before followup CT, underwent surgery, or were under anticoagulant therapy [71].

\section{Coagulation Markers}

D-Dimers are degradation products of fibrinolysis; fibrinogen, an acute phase protein, and Factor XIII participate in blood clot formation. Following ICH their blood concentrations change, as the hemostatic system is activated [75] and might allow prognostic information. 
D-Dimers. In a cohort study, high plasma D-Dimer levels proved a risk factor for 30-day mortality with a sensitivity of $70 \%$ and a specificity of $60 \%$. Moreover, an association between D-Dimer levels and GCS score, midline shift, and subarachnoid extension of the hemorrhage, but no association with intraventricular involvement was shown [76].

Fibrinogen. Fibrinogen was found to be an independent predictor of age, sex, and time from onset to inclusion and Canadian Stroke Scale for early neurologic deterioration $(\mathrm{OR}=5.6)$ in $266 \mathrm{ICH}$ patients [77].

Factor XIII. One study showed increased Factor XIII activities in patients with growing hematomas, and decreased levels in the nongrowing hematoma group [78].

The studies show several associations between coagulation agents and other outcome predictors. Yet at the best cut-off levels, specificity of D-Dimers was rather moderate. Further research in understanding the role of coagulation-related markers in ICH patients is needed.

\section{Copeptin}

Copeptin is the C-terminal part of pro-vasopressin, a neuroendocrine stress marker. As ICH is a major stressor, it activates the neuroendocrine stress axes and since copeptin has been associated with unfavorable outcome in patients with IS, it may also be associated with outcome in ICH patients [79].

Copeptin - measured within $72 \mathrm{~h}$ from symptom onset in a small ICH cohort - was found to positively correlate with hematoma volume and negatively with the GCS. Moreover, high levels of copeptin allowed prediction of 30-day mortality (AUC of 0.88 ) and poor functional outcome at 90 days (AUC of 0.68) [80]. In a larger study, it was shown that copeptin independently predicted 1-year mortality and poor outcome as well as early neurologic deterioration [81].

The studies demonstrate copeptin's potential role as a BBM for risk stratification.

\section{HSP-70}

Heat shock protein 70 (HSP-70) is involved in the process of protein folding and helps to protect cells from stressors including hypoxia or temperature, as occur also in the brain tissue of ICH patients [82].

In a small study, HSP-70 levels measured on the first day after $\mathrm{ICH}$ were found to be higher in nonsurvivors than in survivors [62].

HSP-70 shows some potential as a prognostic marker, but no additive value of HSP-70 to conventional outcome predictors like the Hemphill grading scale have been shown so far.

Blood Biomarkers in Intracerebral Hemorrhage
Plasma Microparticle Concentrations

Microparticles (MPs) are small membrane particles, released by stressed cells under conditions like apoptosis, chemical, or endotoxin stimulation [83]. They seem to play a role in coagulation [84] as well as inflammation [85], both contributing strongly to neuronal damage after $\mathrm{ICH}$.

Plasma MPs independently predicted 7-day mortality with a $91 \%$ sensitivity and $69 \%$ specificity. Plasma MP levels during the first week were higher in patients suffering from intraventricular hemorrhage, in patients with lower GCS scores or greater ICH volumes. Moreover, the study showed that increased MP levels correlated with higher plasma levels of acute phase response markers such as CRP, D-Dimers, fibrinogen, and BGL [86].

Plasma MPs are potential BBMs for the severity of early brain injury after ICH. Interest of future studies should be given to the origin of the measured plasma MPs to enhance our understanding of the cell types involved in cerebral injury.

\section{Selection of Most Interesting Candidates}

\section{Blood Biomarkers Selecting Patients for Specific Treatment Options}

The INTERACT2 study suggests that acute blood pressure-lowering therapies $(<140 \mathrm{~mm} \mathrm{Hg})$ ameliorate outcome for ICH patients [24]. BBMs with promising results addressing early pre-hospital identification of ICH versus IS are GFAP and to some degree S100B/RAGE. If results can be confirmed in larger samples also including mimics and different settings, these BBMs as point-ofcare tools could potentially facilitate early decisions on blood pressure therapy.

It seems interesting to consider whether ICH patients could be further subdivided into groups profiting/not profiting from intensive blood pressure lowering or hemostatic interventions during hospitalization. There are two trials ( $n=339$ [87] and $n=841$ [88]) showing significant reduction of hemorrhage size after the administration of recombinant active factor VII (rFVIIa), but nevertheless fail to demonstrate an overall improved outcome. They propose that patients that were not at risk to experience further expansion of hematoma should have been excluded to result in improved outcomes. Blood levels of several BBMs (e.g., MMP-9, IL-10, see table 2) in ICH show an ability to detect patients at risk for further hemorrhage and might have an importance in selecting patients that would profit from therapies diminishing hematoma growth (table 2). 
Table 2. Biomarkers detecting patients suitable for surgery

Blood biomarkers predicting further bleeding

$\begin{array}{ll}\text { Low LDL } & \text { Associated with hematoma growth at } 24 \mathrm{~h} \\ \text { High Factor XIII } & \text { Found in patients with growing hematomas } \\ \text { High LcC } & \text { Associated with rebleeding within } 30 \text { days } \\ \text { High TNFa and } & \text { Both proved independent predictors for } \\ \text { IL-6 } & \text { early hematoma growth } \\ \text { High IL-10 } & \text { Found to predict rebleeding } \\ \text { High MMP-9 } & \text { Correlates with early hematoma growth }\end{array}$

Also surgical interventions (e.g., hemicraniectomy or minimally invasive procedures) require stability of the patient's condition in regard to further bleeding, to possibly show a better overall outcome [26]. The above-mentioned BBMs that depict risk of further hemorrhagic activities (table 2) might be of use to enroll ICH patients to either medical or surgical intervention. Possibly, a combination of those BBMs might be used for the triage, but so far no study has addressed this specific question.

Only randomized controlled trials comparing algorithms with and without the information of BBM values could answer this question.

\section{Selection of Blood Biomarkers Providing Strong Clinical Evidence \\ Study Design}

As the research interest for BBMs in ICH evolved only over the last decade, the concerning studies reside still in an exploratory phase. Many studies are conducted in a case control design, which bears the problem of selection bias per se. Only few cohort studies, and no randomized controlled trials based on BBMs have been conducted.

\section{Study Population}

The selection of samples and controls is crucial for the interpretation of the data. Current studies of BBMs in ICH often used healthy controls for comparison or they excluded clinically important comorbidities from samples and controls, which most probable - if included would impair the results of the discussed markers. It is therefore remarkable, that the ICH sample for VAP-1/ SSAO didn't exclude systemic diseases (i.e., severe liver disease), although comparing to healthy controls. The marker ApoC-III, on the other hand, is the only case control study, that included orthopedic patients as controls (without any known peripheral/central nervous system condition), and not healthy subjects. The only study differentiating stroke mimics is the cohort for GFAP.

\section{Sample Size}

Also sample size is an important issue and there is clearly need for verification of the results of most discussed BBMs in larger studies. Only few markers were studied in rather large samples, that is, BGL (cohort of 1387 ICH patients), S100B/RAGE (cohort of $139 \mathrm{ICH}$ and 776 IS cases), peripheral LcC (cohort study with 423 ICH patients), fibrinogen (cohort study with $266 \mathrm{ICH}$ patients), CRP (cohort study with $210 \mathrm{ICH}$ cases), and GFAP (cohort study with $39 \mathrm{ICH}, 163$ IS, 3 mimics). However, validation studies in larger multi-center settings are necessary also for these BBMs.

\section{Time from Symptom Onset}

When interpreting the results, it is worth taking into account the particular time of blood withdrawal and the time from symptom onset; both these times should be predefined, as BBMs might follow a dynamic change, therefore altering information or losing comparability over time. BBMs that were measured at multiple predefined time points are S100B, TNFa, IL-11, HSP-70, microparticles, peripheral LcC, ferritin, and GFs, and therefore, allow a more detailed interpretation of the predictive or diagnostic values at given time-points.

\section{Additional Value and Comparison to Clinical \\ Standards}

A new prognostic BBM generally needs to provide additional information to established clinical features like the GCS, the ICH score or imaging data. S100B for example shows a predictive value for 1-week-mortality, that lies slightly (on a not significant level) below the values of the GCS score and ICH volume [58]. An integration to show the additive value to such clinical signs would be interesting, as it has been done for the panel of S100B/RAGE to distinguish between patients suffering from ICH versus IS [35]. In this study, an additive value of the panel to the AUC when added to a model using only clinical signs was significant (AUC 0.84 vs. AUC 0.77). Also HSP-70 was tested in terms of its additive value to conventional outcome predictors, unfortunately with no significant result [62].

\section{Blood Biomarkers Interestingly Linked to \\ Pathophysiology of ICH}

As a BBM, copeptin - the C-terminal of pro-vasopressin - correlates negatively with the GCS, and positively with poor functional outcome, mortality, and hematoma size. A study investigating the inhibition of the arginine vasopressin (AVP) V1 receptor in mice after ICH, could thereby demonstrate a significant reduction in cerebral 
edema formation and significantly less neurobehavioral deficit in the mice treated with the competitive receptor antagonist [89]. They suggest AVP might be a key player of water homeostasis in the brain. Also in a mouse model, VAP-1 (associated with bad outcome in humans) inhibition was shown to reduce brain cell destruction, most likely because it precludes leukocytes from migrating into the injured area, thereby decreasing edema formation [51]. Also s-Fas administration reduced cell-death in a cell model, suggesting a potential therapeutic application of s-Fas [55]. Similarly, another study in rats demonstrated a neuroprotective effect of deferoxamine, an iron chelator, in reducing post ICH edema and neurological injury [90], which underlines the aggrieving role of ferritin in disease progression after $\mathrm{ICH}$, suggesting a potential treatment opportunity of iron chelation in humans. As a $\mathrm{BBM}, \mathrm{BNP}$ correlates with the $\mathrm{ICH}$ score. A recent study showed, that its administration in mice after traumatic brain injury or ICH significantly improved functional outcome [91]. In the mice treated with BNP, inflammatory markers (TNFa, IL-6) decreased, microglia was activated, and neuronal damage was reduced, whereas functional performance and cerebral blood flow were increased [91]. It might be that in more severe ICH cases more BNP is released to improve the recovery, but that the chances of recovery are low due to the initial severity of the ICH. How to translate the effect of BNP in the recovery after ICH in mice to human beings where elevated BNP levels are associated with unfavorable outcome is, however, not yet clear. Such additional results from animal or cell models can add valuable patho-mechanistic information, and inspire further research hypothesis.

\section{Conclusions}

Different approaches and new research, ranging from genomics over proteomics to classical hypothesis-based biomarker identification, on which we focused in this review, suggest novel BBMs. Novel BBMs that correlate with different $\mathrm{ICH}$-related processes might at some point improve the management and outcome of ICH patients. We outlined how each agent is linked to clinical assessment, therefore potentially advancing the current tools for diagnosis, therapy monitoring, risk stratification, or intervention. Nevertheless, for implementation into clinical routine, larger, well-designed prospective studies will be needed to validate findings and prove utility and benefit of the cited BBMs in addition to available treatment protocols and adjuvants like imaging or clinical scores. In this stage of research where large validation studies are needed, effectiveness might be improved by international cooperation and shared resources, such as those provided by the consortium on stroke biomarker research (http:// stroke-biomarkers.com/page.php?title=Resources).

\section{Sources of Funding}

M. Katan received a transatlantic career development award from the foundation Leduqc and a career grant from the Swiss $\mathrm{Na}$ tional Science Foundation (PZ00P3_142422).

\section{Disclosure Statement}

None of the authors state any disclosures.

\section{References}

1 Mukherjee D, Patil CG: Epidemiology and the global burden of stroke. World Neurosurg 2011;76:S85-S90.

$\checkmark 2$ Grysiewicz RA, Thomas K, Pandey DK: Epidemiology of ischemic and hemorrhagic stroke: incidence, prevalence, mortality, and risk factors. Neurol Clin 2008;26:871895 , vii.

- 3 Agnihotri S, Czap A, Staff I, Fortunato G, McCullough LD: Peripheral leukocyte counts and outcomes after intracerebral hemorrhage. J Neuroinflammation 2011;8: 160 .

4 Flaherty ML, Haverbusch M, Sekar P, Kissela B, Kleindorfer D, Moomaw CJ, Sauerbeck L, Schneider A, Broderick JP, Woo D: Longterm mortality after intracerebral hemorrhage. Neurology 2006;66:1182-1186.
5 Manno EM: Update on intracerebral hemorrhage. Continuum (Minneap Minn) 2012;18: 598-610.

6 Lovelock CE, Molyneux AJ, Rothwell PM: Change in incidence and aetiology of intracerebral haemorrhage in Oxfordshire, UK, between 1981 and 2006: a population-based study. Lancet Neurol 2007;6:487-493.

7 Lovelock CE, Cordonnier C, Naka H, Al-Shahi Salman R, Sudlow CL, Sorimachi T, Werring DJ, Gregoire SM, Imaizumi T, Lee SH, Briley D, Rothwell PM: Antithrombotic drug use, cerebral microbleeds, and intracerebral hemorrhage: a systematic review of published and unpublished studies. Stroke 2010;41:1222-1228.

-8 Thanvi BR, Sprigg N, Munshi SK: Advances in spontaneous intracerebral haemorrhage. Int J Clin Pract 2012;66:556-564.
9 Keep RF, Hua Y, Xi G: Intracerebral haemorrhage: Mechanisms of injury and therapeutic targets. Lancet Neurol 2012;11:720-731.

10 Inaji $\mathrm{M}$, Tomita $\mathrm{H}$, Tone $\mathrm{O}$, Tamaki $\mathrm{M}$, Suzuki R, Ohno K: Chronological changes of perihematomal edema of human intracerebral hematoma. Acta Neurochir Suppl 2003;86:445-448.

11 Qureshi AI, Mendelow AD, Hanley DF: Intracerebral haemorrhage. Lancet 2009;373: 1632-1644.

12 Olsson T, Marklund N, Gustafson Y, Nasman B: Abnormalities at different levels of the hypothalamic-pituitary-adrenocortical axis early after stroke. Stroke 1992;23:1573-1576.

13 Sharp F, Liu DZ, Zhan X, Ander BP: Intracerebral hemorrhage injury mechanisms: glutamate neurotoxicity, thrombin, and Src. Acta Neurochir Suppl 2008;105:43-46.
Blood Biomarkers in Intracerebral Hemorrhage 
14 Xi G, Reiser G, Keep RF: The role of thrombin and thrombin receptors in ischemic, hemorrhagic and traumatic brain injury: deleterious or protective? J Neurochem 2003;84:3-9.

-15 Huang FP, Xi G, Keep RF, Hua Y, Nemoianu A, Hoff JT: Brain edema after experimental intracerebral hemorrhage: role of hemoglobin degradation products. J Neurosurg 2002; 96:287-293.

16 Hua Y, Xi G, Keep RF, Hoff JT: Complement activation in the brain after experimental intracerebral hemorrhage. J Neurosurg 2000; 92:1016-1022.

$\checkmark 17$ Hemphill JC 3rd, Bonovich DC, Besmertis L, Manley GT, Johnston SC: The ICH score: a simple, reliable grading scale for intracerebral hemorrhage. Stroke 2001;32:891897.

18 Henley SM, Bates GP, Tabrizi SJ: Biomarkers for neurodegenerative diseases. Curr Opin Neurol 2005; 18:698-705.

19 Rosell A, Vilalta A, Garcia-Berrocoso T, Fernandez-Cadenas I, Domingues-Montanari S, Cuadrado E, Delgado P, Ribo M, MartinezSaez E, Ortega-Aznar A, Montaner J: Brain perihematoma genomic profile following spontaneous human intracerebral hemorrhage. PLoS One 2011;6:e16750.

$>20$ Kodali P, Jurkevica A, Landero J, Kuhlmann C, Caruso J, Adeoye O: Multiple liquid chromatography separations and nanoESI-ion trap detection of plasma proteins in search of stroke biomarkers: a pilot study. J Sep Sci 2012;35:2153-2161.

21 Gilstrap LG, Wang TJ: Biomarkers and cardiovascular risk assessment for primary prevention: an update. Clin Chem 2012;58:7282.

22 Tuntiyatorn L, Saksornchai P, Tunlayadechanont S: Identification of stroke mimics among clinically diagnosed acute strokes. J Med Assoc Thai 2013;96:1191-1198.

-23 Morgenstern LB, Hemphill JC 3rd, Anderson C, Becker K, Broderick JP, Connolly ES Jr, Greenberg SM, Huang JN, MacDonald RL, Messe SR, Mitchell PH, Selim M, Tamargo RJ: Guidelines for the management of spontaneous intracerebral hemorrhage: a guideline for healthcare professionals from the American Heart Association/American Stroke Association. Stroke 2010;41:21082129.

-24 Barber PA, Kleinig TJ: INTERACT2: a reason for optimism with spontaneous intracerebral hemorrhage? Int J Stroke 2014;9:59-60.

25 Bettermann K: Biomarkers for stroke: in search of fingerprints. J Stroke Cerebrovasc Dis 2011;20:173-176.

26 Adeoye O, Broderick JP: Advances in the management of intracerebral hemorrhage. Nat Rev Neurol 2010;6:593-601.

27 Morgenstern LB, Demchuk AM, Kim DH, Frankowski RF, Grotta JC: Rebleeding leads to poor outcome in ultra-early craniotomy for intracerebral hemorrhage. Neurology 2001; 56:1294-1299.
28 Zuccarello M, Brott T, Derex L, Kothari R, Sauerbeck L, Tew J, Van Loveren H, Yeh HS, Tomsick T, Pancioli A, Khoury J, Broderick J: Early surgical treatment for supratentorial intracerebral hemorrhage: a randomized feasibility study. Stroke 1999;30:1833-1839.

29 Prasad V, Bonow RO: The cardiovascular biomarker conundrum: challenges and solutions. JAMA 2011;306:2151-2152.

-30 Missler U, Wiesmann M, Wittmann G, Magerkurth O, Hagenstrom $\mathrm{H}$ : Measurement of glial fibrillary acidic protein in human blood: analytical method and preliminary clinical results. Clin Chem 1999;45:138-141.

31 Dvorak F, Haberer I, Sitzer M, Foerch C: Characterisation of the diagnostic window of serum glial fibrillary acidic protein for the differentiation of intracerebral haemorrhage and ischaemic stroke. Cerebrovasc Dis 2009; 27:37-41.

32 Foerch C, Niessner M, Back T, Bauerle M, De Marchis GM, Ferbert A, Grehl H, Hamann GF, Jacobs A, Kastrup A, Klimpe S, Palm F, Thomalla G, Worthmann H, Sitzer M: Diagnostic accuracy of plasma glial fibrillary acidic protein for differentiating intracerebral hemorrhage and cerebral ischemia in patients with symptoms of acute stroke. Clin Chem 2012;58:237-245.

33 Yan SF, Naka Y, Hudson BI, Herold K, Yan SD, Ramasamy R, Schmidt AM: The ligand/ RAGE axis: lighting the fuse and igniting vascular stress. Curr Atheroscler Rep 2006;8: 232-239.

34 Donato R: Functional roles of S100 proteins, calcium-binding proteins of the EF-hand type. Biochim Biophys Acta 1999;1450:191231.

35 Montaner J, Mendioroz M, Delgado P, Garcia-Berrocoso T, Giralt D, Merino C, Ribo M, Rosell A, Penalba A, Fernandez-Cadenas I, Romero F, Molina C, Alvarez-Sabin J, Hernandez-Guillamon M: Differentiating ischemic from hemorrhagic stroke using plasma biomarkers: the S100B/RAGE pathway. J Proteomics 2012;75:4758-4765.

- 36 Allard L, Lescuyer P, Burgess J, Leung KY, Ward M, Walter N, Burkhard PR, Corthals G, Hochstrasser DF, Sanchez JC: ApoC-I and ApoC-III as potential plasmatic markers to distinguish between ischemic and hemorrhagic stroke. Proteomics 2004;4:2242-2251.

37 Yamada M: Cerebral amyloid angiopathy: an overview. Neuropathology 2000;20:8-22.

38 Hernandez-Guillamon M, Delgado P, Penalba A, Rodriguez-Luna D, Molina CA, Rovira A, Alvarez-Sabin J, Boada M, Montaner J: Plasma beta-amyloid levels in cerebral amyloid angiopathy-associated hemorrhagic stroke. Neurodegener Dis 2012;10: 320-323.

39 Yamada M: Brain hemorrhages in cerebral amyloid angiopathy. Semin Thromb Hemost 2013;39:955-962.

40 Greenberg SM, Cho HS, O’Donnell HC, Rosand J, Segal AZ, Younkin LH, Younkin SG, Rebeck GW: Plasma beta-amyloid pep- tide, transforming growth factor-beta 1 , and risk for cerebral amyloid angiopathy. Ann N Y Acad Sci 2000;903:144-149.

41 Yong VW: Metalloproteinases: mediators of pathology and regeneration in the CNS. Nat Rev Neurosci 2005;6:931-944.

42 Cuadrado E, Rosell A, Penalba A, Slevin M, Alvarez-Sabin J, Ortega-Aznar A, Montaner J: Vascular MMP-9/TIMP-2 and neuronal MMP-10 up-regulation in human brain after stroke: a combined laser microdissection and protein array study. J Proteome Res 2009;8: 3191-3197.

-43 Parks WC, Wilson CL, Lopez-Boado YS: Matrix metalloproteinases as modulators of inflammation and innate immunity. Nat Rev Immunol 2004;4:617-629.

44 Abilleira S, Montaner J, Molina CA, Monasterio J, Castillo J, Alvarez-Sabin J: Matrix metalloproteinase- 9 concentration after spontaneous intracerebral hemorrhage. J Neurosurg 2003;99:65-70.

45 Silva Y, Leira R, Tejada J, Lainez JM, Castillo J, Davalos A: Molecular signatures of vascular injury are associated with early growth of intracerebral hemorrhage. Stroke 2005;36:8691.

46 Wang J, Tsirka SE: Contribution of extracellular proteolysis and microglia to intracerebral hemorrhage. Neurocrit Care 2005;3:7785.

47 Mukoyama M, Nakao K, Hosoda K, Suga S, Saito Y, Ogawa Y, Shirakami G, Jougasaki M, Obata K, Yasue H, et al: Brain natriuretic peptide as a novel cardiac hormone in humans. Evidence for an exquisite dual natriuretic peptide system, atrial natriuretic peptide and brain natriuretic peptide. J Clin Invest 1991; 87:1402-1412.

48 Sviri GE, Soustiel JF, Zaaroor M: Alteration in brain natriuretic peptide (BNP) plasma concentration following severe traumatic brain injury. Acta Neurochir (Wein) 2006;148:529_ 533; discussion 533.

49 James ML, Blessing R, Phillips-Bute BG, Bennett E, Laskowitz DT: S100B and brain natriuretic peptide predict functional neurological outcome after intracerebral haemorrhage. Biomarkers 2009;14:388-394.

50 Hernandez-Guillamon M, Sole M, Delgado P, Garcia-Bonilla L, Giralt D, Boada C, Penalba A, Garcia S, Flores A, Ribo M, Alvarez-Sabin J, Ortega-Aznar A, Unzeta M, Montaner J: VAP-1/SSAO plasma activity and brain expression in human hemorrhagic stroke. Cerebrovasc Dis 2012;33:55-63.

51 Ma Q, Manaenko A, Khatibi NH, Chen W, Zhang JH, Tang J: Vascular adhesion protein-1 inhibition provides antiinflammatory protection after an intracerebral hemorrhagic stroke in mice. J Cereb Blood Flow Metab 2011;31:881-893.

52 Cascino I, Fiucci G, Papoff G, Ruberti G: Three functional soluble forms of the human apoptosis-inducing Fas molecule are produced by alternative splicing. I Immunol 1995;154:2706-2713. 
53 Delgado P, Cuadrado E, Rosell A, AlvarezSabin J, Ortega-Aznar A, Hernandez-Guillamon M, Penalba A, Molina CA, Montaner J: Fas system activation in perihematomal areas after spontaneous intracerebral hemorrhage. Stroke 2008;39:1730-1734.

54 Martin-Villalba A, Hahne M, Kleber S, Vogel J, Falk W, Schenkel J, Krammer PH: Therapeutic neutralization of CD95-ligand and TNF attenuates brain damage in stroke. Cell Death Differ 2001;8:679-686.

-55 Ackery A, Robins S, Fehlings MG: Inhibition of Fas-mediated apoptosis through administration of soluble Fas receptor improves functional outcome and reduces posttraumatic axonal degeneration after acute spinal cord injury. J Neurotrauma 2006;23:604-616.

56 Kim SH, Smith CJ, Van Eldik LJ: Importance of MAPK pathways for microglial pro-inflammatory cytokine IL-1 beta production. Neurobiol Aging 2004;25:431-439.

57 Ponath G, Schettler C, Kaestner F, Voigt B, Wentker D, Arolt V, Rothermundt M: Autocrine $\mathrm{S} 100 \mathrm{~B}$ effects on astrocytes are mediated via RAGE. J Neuroimmunol 2007;184:214222.

$58 \mathrm{Hu}$ YY, Dong XQ, Yu WH, Zhang ZY: Change in plasma $S 100 B$ level after acute spontaneous basal ganglia hemorrhage. Shock 2010;33: 134-140.

59 Lee SH, Kim BJ, Bae HJ, Lee JS, Lee J, Park BJ, Yoon BW: Effects of glucose level on early and long-term mortality after intracerebral haemorrhage: the Acute Brain Bleeding Analysis Study. Diabetologia 2010;53:429-434.

60 Vespa PM: Intensive glycemic control in traumatic brain injury: what is the ideal glucose range? Crit Care 2008;12:175.

-61 Kim JB, Sig Choi J, Yu YM, Nam K, Piao CS, Kim SW, Lee MH, Han PL, Park JS, Lee JK: HMGB1, a novel cytokine-like mediator linking acute neuronal death and delayed neuroinflammation in the postischemic brain. J Neurosci 2006;26:6413-6421.

62 Fang HY, Ko WJ, Lin CY: Inducible heat shock protein 70 , interleukin-18, and tumor necrosis factor alpha correlate with outcomes in spontaneous intracerebral hemorrhage. J Clin Neurosci 2007;14:435-441.

-63 Castillo J, Davalos A, Alvarez-Sabin J, Pumar JM, Leira R, Silva Y, Montaner J, Kase CS: Molecular signatures of brain injury after intracerebral hemorrhage. Neurology 2002;58: 624-629.

64 Wang KW, Cho CL, Chen HJ, Liang CL, Liliang PC, Tsai YD, Wang HK, Lu K: Molecular biomarker of inflammatory response is associated with rebleeding in spontaneous intracerebral hemorrhage. Eur Neurol 2011;66: 322-327.

65 Fang HY, Ko WJ, Lin CY: Plasma interleukin 11 levels correlate with outcome of spontaneous intracerebral hemorrhage. Surg Neurol 2005;64:511-517, discussion 517-518.

-66 Di Napoli M, Godoy DA, Campi V, del Valle M, Pinero G, Mirofsky M, Popa-Wagner A, Masotti L, Papa F, Rabinstein AA: C-reactive protein level measurement improves mortality prediction when added to the spontaneous intracerebral hemorrhage score. Stroke 2011; 42:1230-1236.

67 Zhou Y, Xiong KL, Lin S, Zhong Q, Lu FL, Liang H, Li JC, Wang JZ, Yang QW: Elevation of high-mobility group protein box-1 in serum correlates with severity of acute intracerebral hemorrhage. Mediators Inflamm 2010; pii:142458. DOI: $10.1155 / 2010 / 142458$. Epub 2010 Sep 29.

68 Sobrino T, Arias S, Perez-Mato M, Agulla J, Brea D, Rodriguez-Yanez M, Castillo J: CD34+ progenitor cells likely are involved in the good functional recovery after intracerebral hemorrhage in humans. J Neurosci Res 2011;89:979-985.

69 Sobrino T, Arias S, Rodriguez-Gonzalez R, Brea D, Silva Y, de la Ossa NP, Agulla J, Blanco M, Pumar JM, Serena J, Davalos A, Castillo J: High serum levels of growth factors are associated with good outcome in intracerebral hemorrhage. J Cereb Blood Flow Metab 2009;29:1968-1974.

70 Perez de la Ossa N, Sobrino T, Silva Y, Blanco M, Millan M, Gomis M, Agulla J, Araya P, Reverte S, Serena J, Davalos A: Iron-related brain damage in patients with intracerebral hemorrhage. Stroke 2010;41:810-813.

71 Rodriguez-Luna D, Rubiera M, Ribo M, Coscojuela P, Pagola J, Pineiro S, Ibarra B, Meler P, Maisterra O, Romero F, Alvarez-Sabin J, Molina CA: Serum low-density lipoprotein cholesterol level predicts hematoma growth and clinical outcome after acute intracerebral hemorrhage. Stroke 2011;42:24472452.

72 Roquer J, Rodriguez Campello A, Gomis M, Ois A, Munteis E, Bohm P: Serum lipid levels and in-hospital mortality in patients with intracerebral hemorrhage. Neurology 2005;65: 1198-1202.

73 Noda H, Iso H, Irie F, Sairenchi T, Ohtaka E, Doi M, Izumi Y, Ohta H: Low-density lipoprotein cholesterol concentrations and death due to intraparenchymal hemorrhage: the Ibaraki Prefectural Health Study. Circulation 2009;119:2136-2145.

74 Ramirez-Moreno JM, Casado-Naranjo I, Portilla JC, Calle ML, Tena D, Falcon A, Serrano A: Serum cholesterol LDL and 90-day mortality in patients with intracerebral hemorrhage. Stroke 2009;40:1917-1920.

75 Fujii Y, Takeuchi S, Harada A, Abe H, Sasaki O, Tanaka R: Hemostatic activation in spontaneous intracerebral hemorrhage. Stroke 2001;32: 883-890.

76 Chiu CC, Li YN, Lin LJ, Hsiao CT, Hsiao KY, Chen IC: Serum D-dimer as a predictor of mortality in patients with acute spontaneous intracerebral hemorrhage. J Clin Neurosci 2012;19:810-813.

-77 Leira R, Davalos A, Silva Y, Gil-Peralta A, Tejada J, Garcia M, Castillo J: Early neurologic deterioration in intracerebral hemorrhage: predictors and associated factors. Neurology 2004;63:461-467.
78 Marti-Fabregas J, Borrell M, Silva Y, DelgadoMederos R, Martinez-Ramirez S, de JuanDelago M, Tirado I, Alejaldre A, Marin R, Marti-Vilalta JL, Fontcuberta J: Hemostatic proteins and their association with hematoma growth in patients with acute intracerebral hemorrhage. Stroke 2010;41:2976-2978.

79 Katan M, Fluri F, Morgenthaler NG, Schuetz P, Zweifel C, Bingisser R, Muller K, Meckel S, Gass A, Kappos L, Steck AJ, Engelter ST, Muller B, Christ-Crain M: Copeptin: a novel, independent prognostic marker in patients with ischemic stroke. Ann Neurol 2009;66:799-808.

80 Zweifel C, Katan M, Schuetz P, Siegemund M, Morgenthaler NG, Merlo A, Mueller B, ChristCrain M: Copeptin is associated with mortality and outcome in patients with acute intracerebral hemorrhage. BMC Neurol 2010;10:34.

81 Zhang X, Lu XM, Huang LF, Ye H: Copeptin is associated with one-year mortality and functional outcome in patients with acute spontaneous basal ganglia hemorrhage. Peptides 2012;33:336-341.

\$2 Kim JY, Yenari MA: The immune modulating properties of the heat shock proteins after brain injury. Anat Cell Biol 2013;46:1-7.

83 Nomura S, Ozaki Y, Ikeda Y: Function and role of microparticles in various clinical settings. Thromb Res 2008;123:8-23.

84 Ardoin SP, Shanahan JC, Pisetsky DS: The role of microparticles in inflammation and thrombosis. Scand J Immunol 2007;66:159-165.

-85 Distler JH, Huber LC, Gay S, Distler O, Pisetsky DS: Microparticles as mediators of cellular cross-talk in inflammatory disease. Autoimmunity 2006;39:683-690.

86 Dong XQ, Huang M, Hu YY, Yu WH, Zhang ZY: Time course of plasma microparticle concentrations after acute spontaneous basal ganglia hemorrhage. Acta Neurol Scand 2011; 123:280-288.

-87 Mayer SA, Brun NC, Begtrup K, Broderick J, Davis S, Diringer MN, Skolnick BE, Steiner T: Recombinant activated factor VII for acute intracerebral hemorrhage. $\mathrm{N}$ Engl $\mathrm{J}$ Med 2005;352:777-785.

88 Mayer SA, Brun NC, Begtrup K, Broderick J, Davis S, Diringer MN, Skolnick BE, Steiner T: Efficacy and safety of recombinant activated factor VII for acute intracerebral hemorrhage. N Engl J Med 2008;358:2127-2137.

89 Manaenko A, Fathali N, Khatibi NH, Lekic T, Hasegawa Y, Martin R, Tang J, Zhang JH: Arginine-vasopressin V1a receptor inhibition improves neurologic outcomes following an intracerebral hemorrhagic brain injury. Neurochem Int 2011;58:542-548.

90 Song S, Hua Y, Keep RF, Hoff JT, Xi G: A new hippocampal model for examining intracerebral hemorrhage-related neuronal death: effects of deferoxamine on hemoglobin-induced neuronal death. Stroke 2007;38:2861-2863.

91 James ML, Wang H, Venkatraman T, Song P, Lascola CD, Laskowitz DT: Brain natriuretic peptide improves long-term functional recovery after acute CNS injury in mice. J Neurotrauma 2010;27:217-228.
Blood Biomarkers in Intracerebral Hemorrhage
Cerebrovasc Dis 2014;38:395-409 DOI: $10.1159 / 000366470$ 